\title{
Fault-Tolerant Model Predictive Control of Hybrid Systems
}

\author{
Yosra Hammi, Nadia Zanzouri, Mekki Ksouri \\ Unity of Research Analysis and Control of Systems (ACS) \\ National School of Engineering of Tunis \\ BP, 37, Le Belvédère, 1002 Tunisia
}

\begin{abstract}
This paper, focuses on model predictive control (MPC) problem with fault-tolerance capabilities is formulated within the hybrid systems framework In particular Mixed Logical Dynamical (MLD) approach is considered. It allows to model the hybrid systems involved continuous, discrete dynamics and constraints. The changes or the switches which may appear over such dynamics are modeled by using the auxiliary variables taking into account the interconnections. In this work, we proposed a reconfiguration control approach based on Model Predictive Control (MPC) framework. A fault MLD model is also proposed. The main contribution of this paper consists in the investigation of a new method for fault tolerant control used the MLD model. The proposed formulation is illustrated by considering a two tank system benchmark.
\end{abstract}

\section{Keywords}

Hybrid System, MLD, Control Reconfiguration, actuators Faults, MLDF, MPC.

\section{INTRODUCTION}

Many control systems are subject to faults or malfunctions. The failure of components, like sensors or actuators can partially or fully disable the action of the controller. This results in an unsatisfactory performance of the system, decreased availability, emergency shutdowns or even significant damages to the plant. In addition. In modern technological systems, there is a high demand on performance, safety, and reliability of systems. It is desired that if a fault happens, the control system can automatically detect the fault and moderate its effect on the system such that it can continue working while providing an acceptable performance.

In any case, it is important to avoid dangerous areas to prevent damages to the system. Therefore, Fault tolerance control is very important for modern technological systems.

In fact, the fault Tolerant systems in literature can be derived into two main groups: active and passive techniques. On the one hand, the passive technique is designed, such that it is robust, within performance range, to fault occurrences. On the other hand, the active fault tolerant system aims at changing the control operation when the fault is detected. These changes can comprise reconfiguration of the controller scheme, modification of controller parameters or alternative set point trajectories. In recent decades there exists an emerging area of research working in Fault tolerant control of hybrid systems, for a survey one can look at [1] [2], [3]. A class of approaches for diagnosis of hybrid systems discrete/temporal abstraction of the continuous dynamics is presented in [4]. In [5], the authors use a Petri net abstraction for dealing with continuous behaviors of hybrid systems. In [6], the diagnose uses a discrete event abstraction of the system and the continuous dynamics information is taken in consideration when it becomes necessary. In [3] a model based diagnosis method on a hybrid bond graph modelling framework is proposed. Motivated by different capabilities and applications of the mixed logical dynamical (MLD) modeling of hybrid system, many approaches have been reported in [7], [8], [9]. Moreover using the MLD framework problems such as optimal control state estimation,. This can be reformulated as a mixed integer programming problem and therefore solved using the same technique. This paper, deals with MLD formalism First, we proposed a new formulation integrating the faults in MLD model. The MPC controller is investigated for control objective. The aim of this paper consists in embedding the active fault-tolerant design of controllers based on model predictive control (MPC) within the hybrid system framework. In this context, a new methodology is developed. The goal is to verify the fault tolerance capabilities of MPC and computational aspects of MLDF framework to deal with hybrid systems modeling and control problem. The paper is organized as follows: in section 2, we introduce basic propositional logic rules, Boolean algebra, and mixed-integer linear inequalities. These tools are used to define the MLD formalism. Predictive control of MLD model is developed in section 3.Different steps followed to obtain the MLD faults model we proposed in section 4. In addition MPC with MLD faults are study also the problem of active diagnosis is formulated and solution is proposed. Simulation results are presented and discussed in section 5 . The paper is concluded in section 6 .

\section{MIXED LOGIC DYNAMICAL SYSTEMS MODELLING}

Mixed logical dynamical formalism is a powerful modeling approach in hybrid systems theory. It transforms dynamics, logic and constraints of a complex 
system into an integrated model, logical and dynamical constraints are translated to mixed-integer inequalities [9]-[10]

Mixed-integer inequalities contain continuous and integer variables. In fact using binary and auxiliary variables, hybrid systems are modeled as linear dynamical systems with mixed-integer inequalities constraints. Mixed logical dynamical modeling allows the state and the control inputs to be continuous or discrete. A binary variable is associated with each logical relation like:

$$
X_{i}=\text { True } \leftrightarrow \delta_{i}=1
$$

Propositional logic is also translated to equivalent linear inequalities. As an example can be shown that:

$X_{1} \vee X_{2}$ is equivalent to $\delta_{1}+\delta_{2} \geq 1$.

Another basic principle of MLD modeling is the interaction between logical and dynamical variables. It can be proved that $[\delta=1] \leftrightarrow[f(x) \leq 0]$ is true if

$$
\left\{\begin{array}{l}
f(x) \leq M-M \delta \\
f(x) \geq \varepsilon+(m-\varepsilon) \delta
\end{array}\right.
$$

Where $\mathrm{M}(\mathrm{m})$ is maximum (minimum) or an upper (lower) bound of $f$ and $\varepsilon$ is a small positive number.

This equivalence permits the assignment of binary variables to dynamical constraints which may define the different operation modes of hybrid system. Another useful equivalence that deals with the interaction of logical and dynamical variables is as follows: The equality relation $z=\delta f(x)$ regardless of the relation between $\delta$ and $f(x)$ could be translated to the following four mixed integer inequalities:

$$
\left\{\begin{array}{l}
z \leq M \delta \\
z \geq m \delta \\
z \leq f(x)-m(1-\delta) \\
z \geq f(x)-M(1-\delta)
\end{array}\right.
$$

In fact the MLD modeling framework [9], is based on the idea of translating logic relations, discrete/logic dynamics, A/D (analog to digital (logic)), D/A conversion and logic constraints into mixed integer linear inequalities. These inequalities are combined with the continuous dynamical part, which are described by linear difference equations. The resulting MLD system is described by the following relations [9]. $\left\{\begin{array}{l}x(k+1)=A x(k)+B_{1} u(k)+B_{2} \delta(k)+B_{3} z(k) \\ y(k)=C x(k)+D_{1} u(k)+D_{2} \delta(k)+D_{3} z(k) \\ E_{2} \delta(k)+E_{3} z(k) \leq E_{1} u(k)+E_{4} x(k)+E_{5}\end{array}\right.$

Where:

$$
A, B_{1}, B_{2}, B_{3}, C, D_{1}, D_{2}, D_{3}, E_{1}, E_{2}, E_{3}, E_{4} \text { and } \quad E_{5}
$$
are matrices of appropriate dimension.

The continuous and binary (mixed) variables $\mathrm{x}, \mathrm{y}$ and $\mathrm{u}$ are respectively the state, inputs and outputs of MLD system which are defined as follows:

$$
\begin{aligned}
& x=\left[\begin{array}{l}
x_{c} \\
x_{l}
\end{array}\right], x_{c} \in \mathfrak{R}^{n_{c}}, x_{l} \in\{0,1\}^{n_{l}}, x \in \mathfrak{R}^{n}, n=n_{c}+n_{l} \\
& y=\left[\begin{array}{l}
y_{c} \\
y_{l}
\end{array}\right], y_{c} \in \mathfrak{R}^{p_{c}}, y_{l} \in\{0,1\}^{p_{l}}, y \in \mathfrak{R}^{p}, p=p_{c}+p_{l} \\
& u=\left[\begin{array}{l}
u_{c} \\
u_{l}
\end{array}\right], u_{c} \in \mathfrak{R}^{m_{c}}, u_{l} \in\{0,1\}^{m_{l}}, u \in \mathfrak{R}^{m}, m=m_{c}+m_{l}
\end{aligned}
$$

The variables $\delta$ and $z$ are introduced when translating logic propositions into linear inequalities.

$\delta(k) \in\{0,1\}^{r_{l}}$ are the auxiliary binary variables, which represent the events in the hybrid dynamical system.

$z(k) \in \mathfrak{R}^{r_{c}}$ are the auxiliary continuous variables, which combination continuous dynamics and discrete dynamics. The variables $\delta$ and $z$ are introduced when translating logic propositions into linear inequalities. There are used to define the relations between continuous and discrete parts.

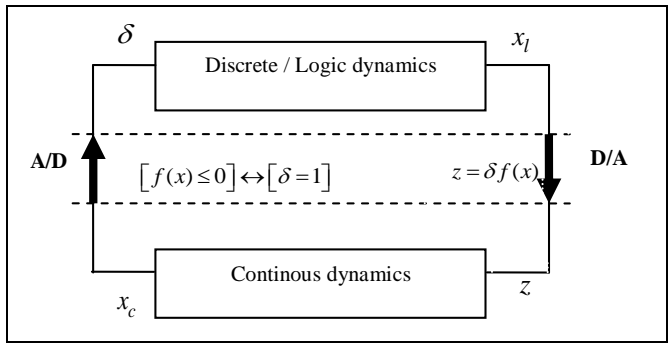

Fig 1: MLD structure

\section{Academic example}

In this section, an illustrating example is considered [9]. The procedure of MLD modeling is applied to this system described by the following equations:

$$
x(k+1)=\left\{\begin{array}{lr}
0.3 x(k)+u(k) & \text { si } x(k) \geq 0 \\
-0.8 x(k)+u(k) & \text { si } x(k)<0
\end{array}\right.
$$

Where, $x(k) \in\left[\begin{array}{ll}-10 & 10\end{array}\right]$ and $u(k) \in\left[\begin{array}{ll}-1 & 1\end{array}\right]$ To construct the MLD model, the condition $x(k) \geq 0$ can be associated to a binary variable $\delta(\mathrm{k})$ such that

$$
x(k) \geq 0 \leftrightarrow \delta(k)=1
$$

By using the transformations described the equation (2) can be expressed by the following inequalities:

$$
\left\{\begin{array}{l}
-x(k) \leq-m(1-\delta(k)) \\
x(k) \leq-\varepsilon+\delta(k)(M+\varepsilon)
\end{array}\right.
$$

where $M=-m=10$, and $\varepsilon$ is a small positive scalar. Then, equation (5) can be rewritten as: 


$$
x(k+1)=1.1 \delta(k) x(k)-0.8 x(k)+u(k)
$$

By defining a new variable $z(k)=\delta(k) x(k)$, using the equation (3) this auxiliary variable is expressed as follows:

$$
\left\{\begin{array}{l}
z(k) \leq 10 \delta(k) \\
z(k) \geq-10 \delta(k) \\
z(k) \leq x(k)+10(1-\delta(k)) \\
z(k) \geq x(k)-10(1-\delta(k)
\end{array}\right.
$$

So the system (8) is ruled by this linear equation: $x(k+1)=1.1 z(k)-0.8 x(k)+u(k)$

Subject to the linear constraints (7) and (9). Therefore, the MLD equivalent representation of (5) for $x \in[-10,10]$ and $u \in[-1,1]$ is given by collecting Equations (7), (9) and (10).

\section{PREDICTIVE CONTROL BASED ON MLD MODEL}

The main idea of MPC is to use a model of the system to predict the future evolution of the system in a fixed prediction horizon with the measurements of the system. Based on this prediction at each time step $\mathrm{k}$, the controller selects a sequence of future command inputs through an optimization procedure, which aims at minimizing a suitable cost function and enforces fulfilment of the constraints. Then, only the first sample of the optimal sequence is applied to the plant at time step $\mathrm{k}$, and at time step $\mathrm{k}+\mathrm{l}$, the whole optimization procedure is repeated with new plant measurements this online replanning provides the desired feedback control action. Let $\mathrm{k}$ be the current time step, $\mathrm{Hp}$ is the prediction horizon, and $\mathrm{x}(\mathrm{k})$ is the current state.

An important control problem for MLD systems is to stabilize the system to an equilibrium state or to track a desired reference trajectory. In general finding a control law that attains these objectives for an MLD system is not an easy task, as in general MLD systems are neither linear nor even smooth. MPC provides a successful tool to perform this task, as will be shown next. For the sake of brevity we will concentrate on the stabilization to an equilibrium state.

In fact, the following theorem [11] show that MPC problem, feasibility is preserved over time and that feasibility implies stability.Consider the MLD system (4) and an equilibrium state/input/output triple $\left(x_{e q}, u_{e q}, y_{e q}\right)$ and let $\left(\delta_{e q}, z_{e q}\right)$ be the corresponding pair of auxiliary variables.

Assume that the initial state $x(0)$ is such that a feasible solution of the MLD-MPC problem exists for sample step 0 . The input signal resulting from applying the optimal MLD-MPC input signal in a receding horizon approach stabilizes the MLD system in the sense that

$$
\begin{array}{ll}
\lim _{k \rightarrow \infty} x(k)=x_{e q}, & \lim _{k \rightarrow \infty}\left\|y(k)-y_{e q}\right\|_{Q_{y}}=0, \\
\lim _{k \rightarrow \infty} u(k)=u_{e q}, & \lim _{k \rightarrow \infty}\left\|\delta(k)-\delta_{e q}\right\|_{Q_{\delta}}=0, \\
& \lim _{k \rightarrow \infty}\left\|z(k)-z_{e q}\right\|_{Q_{z}}=0 .
\end{array}
$$

This problem can be solved using powerful mixed integer quadratic programming (MIQP) algorithm [9].

In fact, for system modeled with MLD formalism the optimization problem of predictive control MPC has the following form:

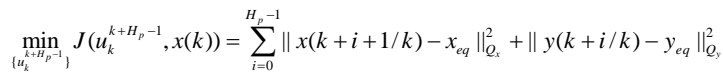

$$
\begin{aligned}
& +\left\|u(k+i / k)-u_{e q}\right\|_{Q_{u}}^{2}+\left\|\delta(k+i / k)-\delta_{e q}\right\|_{Q_{s}}^{2}+\left\|z(k+i / k)-z_{e q}\right\|_{Q_{:}}^{2}
\end{aligned}
$$

Where $Q_{u}, Q_{x}$ are positive definite matrices, and

$Q_{y}, Q_{\delta}, Q_{z}$ are nonnegative definite matrices. Furthermore, the MLD system equations we have the end-point condition $x\left(H_{p} \mid k\right)=x_{e q}$ (stability constraint). In addition, the optimal MPC minimizes the objective function $J$ subject to constraints:

$$
\left\{\begin{array}{l}
x(0 \mid k)=x(k) \\
x\left(H_{p} \mid k\right)=x_{e q} \\
x(i+1 \mid k)=A x(i \mid k)+B_{1} u(i)+B_{2} \delta(i \mid k)+B_{3} z(i \mid k) \\
y(i \mid k)=C x(i \mid k)+D_{1} u(i)+D_{2} \delta(i \mid k)+D_{3} z(i \mid k) \\
E_{2} \delta(i \mid k)+E_{3} z(i \mid k) \leq E_{1} u(i)+E_{4} x(i \mid k)+E_{5}
\end{array}\right.
$$

We assume that there exists an optimal sequence $u_{k}^{*} \square\left\{u^{*}(0), u^{*}(1), \ldots, u^{*}(N-1)\right\}$ for this problem. The MPC control law is defined as the first element of this sequence $u^{*}(0)$. The input is applied to the system and the whole procedure is repeated at the next time instance.

\section{ACTIVE FAULT TOLERANT CONTROL USING MPC AND MLD FORMALISM}

In this section, presented a methodology ways including fault tolerance in MPC controller. According to Maciejowski [12] [13] when using an MPC controller, if the knowledge of faults is available, either the internal model or system constraints can be modified accordingly. In this way, fault tolerance can be implicitly incorporated into an MPC controller in a natural way. Furthermore, due to the flexibility for expressing the control objectives within the MPC formalism, when faults cause control objectives to become unattainable, they can be dropped from the optimization problem or degraded in priority, for example, by changing hard constraints to soft ones . In fact, having the model define in 4 contains continuous and binary input and outputs. I will be developed a methodology for integrated faults in MLD model.

\subsection{Formulation of MLDF (Mixed logical dynamical faults)}

$>$ Faults actuator or sensor

The faults actuator or sensor is presented by:

$$
\begin{aligned}
& u_{\text {app }}=\left(1-\alpha_{u}\right) u_{c}=u_{c}-u_{f c} \\
& y_{\text {mes }}=\left(1-\alpha_{y}\right) y_{c}=y_{c}-y_{f c}
\end{aligned}
$$

With $\alpha_{y}, \alpha_{u} \in[0,1]$ 
If $\alpha_{u}, \alpha_{y}=0$ no failure

If $\alpha_{u}, \alpha_{y}=1$ total loss of actuator or sensor

$\alpha_{u}, \alpha_{y}$ : Coefficients of failure.

Where $u_{\text {app }}:$ applied control system

$$
\begin{aligned}
& u_{c}: \text { computed control system } \\
& u_{f c}: \text { continuous failure control } \\
& y_{m e s}: \text { output sensor }
\end{aligned}
$$$$
y_{c} \text { : measured output with the normal operation }
$$
sensor

$$
y_{f_{c}} \text { : continuous sensor fault }
$$

For obtain the MLD fault, the key idea is to model the effects of a fault on the system as logic proposition and to translate them into inequalities to be added to the MLD model of a system. In fact, for presented actuator faults or sensor faults in MLD model, we need to introduce the auxiliary continuous variables zf. For sensor faults:

$$
\begin{gathered}
y_{\text {mes }}=\left(1-\alpha_{y}\right) y_{c}=y_{c}-z_{f} \\
z_{f}=\delta_{f} \cdot y_{f_{c}} \quad \delta_{f} \in\{0,1\}
\end{gathered}
$$

Therefore, two cases can arise if:

$$
\begin{array}{cc}
\delta_{f}=0 \rightarrow z_{f}=0 & \text { no fault } \\
\delta_{f}=1 \rightarrow z_{f}=y_{f_{c}} & \text { fault }
\end{array}
$$

In fact the addition of continuous auxiliary variable leads to increased inequality constraints as an auxiliary variable can be converted into linear inequalities following 4 equations

$$
z_{f}=\delta_{f} \cdot y_{f c}\left\{\begin{array}{l}
z \leq M \delta \\
z \geq m \delta \\
z \leq f(x)-m(1-\delta) \\
z \geq f(x)-M(1-\delta)
\end{array}\right.
$$

with $m=\min \left(y_{f_{c}}\right), M=\max \left(y_{f c}\right)$

We can define the MLD fault as follow:

$$
\begin{aligned}
& x(k+1)=A x(k)+B_{1} u(k)+B_{2} \tilde{\delta(k)}+B_{3} z(\tilde{k}) \\
& \left.y(k)=C x(k)+D_{1} u(k)+D_{2} \tilde{\delta} \tilde{k}\right)+D_{3} z(\vec{k}) \\
& \tilde{E}_{2} \delta \tilde{(k)}+\tilde{E}_{3} z \tilde{(k)} £ \tilde{E}_{1} u(k)+\tilde{E}_{4} x(k)+\tilde{E}_{5}
\end{aligned}
$$

$$
\text { With } \tilde{\delta}=\left[\begin{array}{l}
\delta \\
\delta_{f}
\end{array}\right], \quad \tilde{z}=\left[\begin{array}{c}
z \\
z_{f}
\end{array}\right]
$$

Finally, we obtain the MLD fault including the normal and failure mode and all constraints of the systems

\subsection{Formulation problem and solution proposed}

A standard control [14] problem can be shown by the following triple: $\langle\mathrm{O}, C(\Theta), U\rangle$

where:

\section{O Global objectives}

$C(\Theta)$ Structure of system which depend parameters $\Theta$

$U$ Control law

Solving this problem consists in finding a control $u \in U$ so as to achieve the global objective $\mathrm{O}$ under constraints whose structure $C(\Theta)$.

In the fault free case the nominal global objectives $\mathrm{O}$ are assumed to be achieved under the nominal control $U_{n}$ land nominal constraint structure $C\left(\Theta_{n}\right)$.

The occurrence of faults can modify the structure of the system, meaning that global objectives can be or not achieved under the new structure.

\section{Solution proposed}

A new formulation of the problem $\left\langle\mathrm{O}, C\left(\Theta_{f}\right), U_{f}\right\rangle$ is proposed, which has a solution and thus allows achieving $\mathrm{O}$, by changing the system structure, using the model system proposed MLD Fault and $\operatorname{control} U_{f}$. (which result from the disconnection or replacement of fault components). In some cases, no solution exists, and then global objectives must be redefined to degraded ones.. In our case, the FDI module figure 2 functionality is assumed to work correctly. Ideally it detects and isolates the faulty also the fault information is assumed readily available and is used to modify the corresponding constraints in the optimization problem (19).

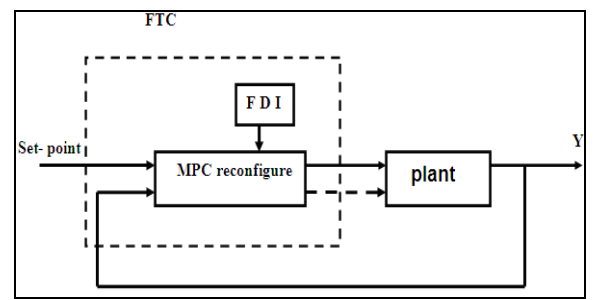

Fig2: MPC Fault Tolerant Controller

In fact, for including the faulty mode in MLD model of the system, we need to generate additional binary variable corresponding to faulty mode (zf). If the fault is detected, the active fault tolerant control technique consists to reconfigure the model predictive controller for 
the faulty system by changing the constraints to reflect the identified fault. Therefore, the MPC problem should be modified accordingly by replacing the MLD by $\operatorname{MLDF}(17)$.

In faulty mode, the criteria $J(k)$ is modified as fellow:

$$
\begin{gathered}
\min _{\left\{u_{k}^{k+p_{p}-1}\right\}} J\left(u_{k}^{k+H_{p}-1}, x(k)\right)=\sum_{i=0}^{H_{p}-1}\left\|x(k+i+1 / k)-x_{r f}\right\|_{Q_{x}}^{2} \\
+\left\|y(k+i / k)-y_{r f}\right\|_{Q_{y}}^{2}+\left\|u(k+i / k)-u_{r f}\right\|_{Q_{u}}^{2} \\
+\left\|\delta(k+i / k)-\delta_{r f}\right\|_{Q_{j}}^{2}+\left\|z(k+i / k)-z_{r f}\right\|_{Q_{z}}^{2}
\end{gathered}
$$

and we note in this case another optimization problem MIQP under constraints (20) and another equilibrium state must be found for the faulty system.

$$
\left\{\begin{array}{l}
x(0 \mid k)=x(k) \\
x_{r f}=x\left(H_{p} \mid k\right) \\
x(i+1 \mid k)=A x(i \mid k)+B_{1} u(i)+B_{2} \tilde{\delta}(i \mid k)+B_{3} \tilde{z}(i \mid k) \\
y(i \mid k)=C x(i \mid k)+D_{1} u(i)+D_{2} \tilde{\delta}(i \mid k)+D_{3} \tilde{z}(i \mid k) \\
E_{2} \tilde{\delta}(i \mid k)+E_{3} \tilde{z}(i \mid k) \leq E_{1} u(i)+E_{4} x(i \mid k)+E_{5}
\end{array}\right.
$$

It means, the system is fault-tolerant with respect to the fault $\mathrm{f}$ and the control objective $\mathrm{O}$, if there exist a solution to the above problem. If the system is not faulttolerant, a possible solution is to change the control objective, e.g. by allowing some degradation in the performance of the system or by just considering the stability of the closed loop system.

\section{ILLUSTRATIVE SYSTEM}

\subsection{Two tank system}

The benchmark of the two tank system has been used in many fields of research to illustrate the work realized in the CNRS research group especially in AS 193 diagnosis and hybrid systems [15]. The system consists of two liquid tanks that can be filled with pump acting on the tank1 (figure 3). The pump P1 delivers the liquid flows Qp1 and it can be manipulated from a zero flow to a maximum flow Qmax. The tanks are interconnected by two pipes at the bottom and at level hv. The flows through the pipes are denoted by Q4, V4 and Q3, V3. The flow through these pipes can be interrupted with switching valve V4. The liquid levels $h 1$ and $h 2$ in each tank can be measured with continuous level sensors. The two discrete inputs considered: are the state of valve V2 and V4.

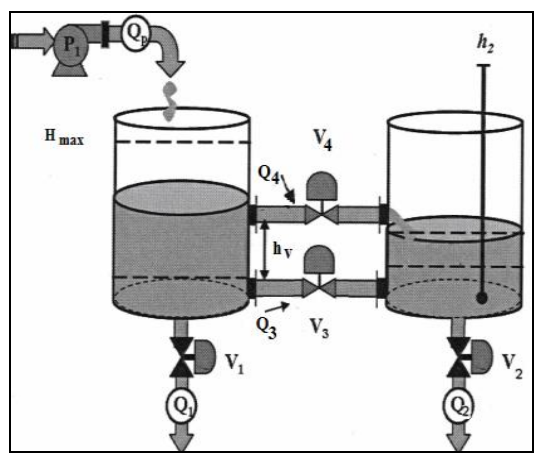

Fig3: Two tank system
From the conservation mass of law balance tanks, we obtain the following differential equation

$$
\dot{V}=A \dot{h}=\sum Q_{\text {in }}-\sum Q_{\text {out }}
$$

Where $Q_{\text {in }}$ is the sum of input flow and $Q_{\text {out }}$ is the sum of output flows, A is the section of the tank, $V$ is the volume of liquid in the tank and $h$ is the level liquid. We obtain:

$$
\begin{aligned}
& \left\{\begin{array}{l}
\dot{h}_{1}=\frac{1}{A}\left(Q_{p 1}-Q_{1} V_{1}-Q_{3} V_{3}-Q_{4} V_{4}\right) \\
\dot{h}_{2}=\frac{1}{A}\left(Q_{3} V_{3}+Q_{4} V_{4}-Q_{2} V_{2}\right)
\end{array}\right. \\
& Q_{1} V_{1}=V_{1} S \sqrt{2 g} \sqrt{h_{1}} \\
& Q_{2} V_{2}=V_{2} S \sqrt{2 g} \sqrt{h_{2}} \\
& Q_{3} V_{3}=V_{3} S \operatorname{sign}\left(h_{1}-h_{2}\right) \sqrt{2 g} \sqrt{\left|h_{1}-h_{2}\right|} \mathrm{A} \\
& Q_{4} V_{4}=V_{4} S \sqrt{2 g} \sqrt{\left|\max \left(h_{1}, 0.5\right)-\max \left(h_{2}, 0.5\right)\right|}
\end{aligned}
$$

The nonlinear relation $\sqrt{x}$ is approximated by a straight line $\mathrm{x}$, thus becomes:

$$
Q_{3} V_{3}=V_{3} k_{3}\left(h_{1}-h_{2}\right)
$$

The auxiliary continuous variable $z_{3}=V_{3}\left(h_{1}-h_{2}\right)$ is introduced to transform the above nonlinear equation to the linear equation $Q_{3} V_{3}=k_{3} z_{3}$ with a set of mixed integer linear inequalities. For $Q_{1}$ and $Q_{2}$, using the same method, we will have $Q_{1}=k_{1} z_{1}$ and $Q_{2}=k_{2} z_{2}$ where $z_{1}=h_{1} V_{1}$ and $z_{2}=h_{2} V_{2}$ for more detail see [16].

In order to take into account the flows through the upper valve V4, we define the auxiliary binary variables indicating whether the level in each tank has reached hv:

$$
\begin{array}{ll}
{\left[\delta_{1}(k)=1\right]} & \leftrightarrow\left[h_{1}(k) \geq h_{v}\right] \\
{\left[\delta_{2}(k)=1\right]} & \leftrightarrow\left[h_{2}(k) \geq h_{v}\right]
\end{array}
$$

and then the term $\max \left(h_{1}, 0.3\right)-\max \left(h_{2}, 0.3\right)$ is transformed into a linear equation $Q_{4} V_{4}=k_{4} z_{4}$

where:

$$
\left\{\begin{array}{l}
z_{4}=V_{4}\left(z_{01}-z_{02}\right) \\
z_{01}=\delta_{1}\left(h_{1}-h_{v}\right) \\
z_{02}=\delta_{2}\left(h_{2}-h_{v}\right)
\end{array}\right.
$$

are auxiliary continuous variables.

Finally, the differential equations (6) are discretized by using the Euler method by replacing $\dot{h}$ with the $\frac{h(k+1)-h(k)}{T_{S}}$ where $T_{S}$ is the sampling time.

Let first defining the state vector variables $x \square\left[h_{1} h_{2}\right]^{T}$ 
The input vector $u \square\left[Q_{1} V_{4} V_{2}\right]^{T}$ combined a continuous part and discrete part.

$\delta \square\left[\delta_{1} \delta_{2}\right]^{T}$ are the auxiliary binary variables .

$z \square\left[z_{01} z_{02} z_{1} z_{2} z_{3} z_{4}\right]^{T}$ are the auxiliary continuous variables modelled the changes or the switches which may appear over such dynamics.

The transformation of the hybrid system equations into the MLD form requires the application of a set of given rules. A higher level language and associated compiler (HYSDEL, see [17-18] are used here to avoid the tedious procedure of deriving the MLD form by hand. Given the MLD model, the scenarios are simulated using the Hybrid Toolbox for Matlab [19].

\subsection{Simulation and Results}

The proposed method is tested on two tank system. In order to show the capability of handling a multivariable hybrid control problem we have simulated the system (figure.4) combining pumps and switching valves. The results of the predictive control are shown in Figure 5. As can be seen the states reaches to the given reference signal h1 and h2.

$$
0 \leq Q \leq 1.10^{-4} m^{3} . s^{-1} \quad 0 \leq h_{i} \leq 0.8 m
$$

In the first case, we have considered a constant reference having 0.6 and 0.3 value Figure 4, but in the second one a variable reference having sinusoidal form has been taken Figure 6.
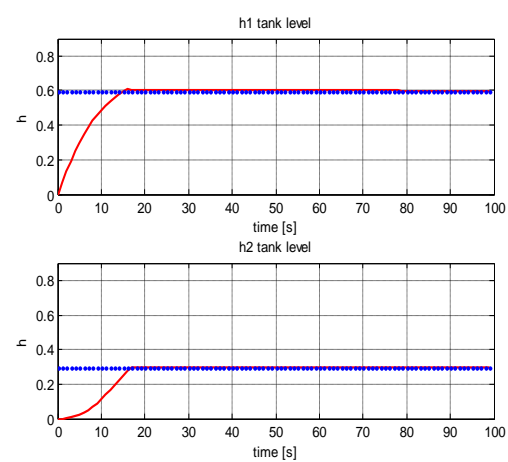

Fig4: Liquid level in the two tank system

We see that the water level does not exceed the maximum level already imposed $(\mathrm{hmax}=0.8 \mathrm{~m})$. The flow of pump figure 7 also does not exceed ( $\mathrm{Qmax}=1$ 10-4m3/s).

Moreover if we change the reference, which is a critical case we also obtain an acceptable control result in this new given condition figure (6).
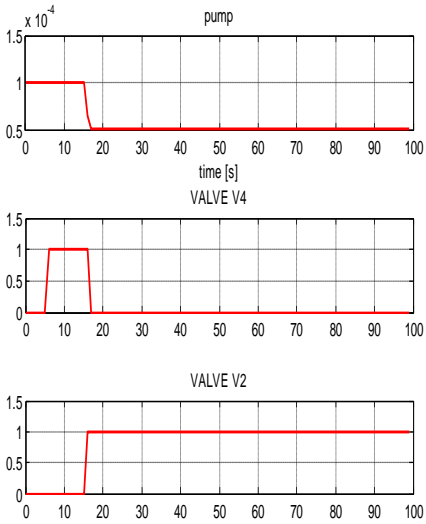

Fig5: Continuous input Qp1, discrete inputs V4 and V2

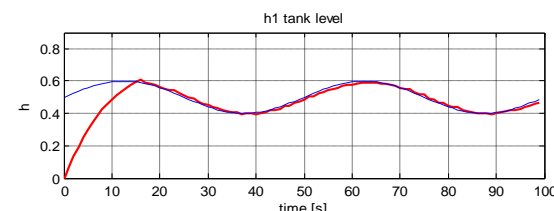

time $[s]$
h2 tank leve

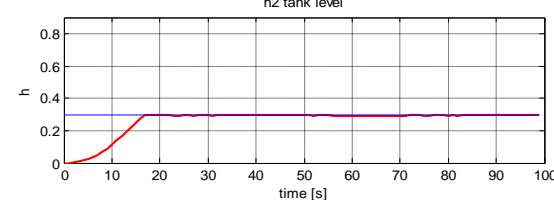

Fig 6: Liquid level in the two tank system

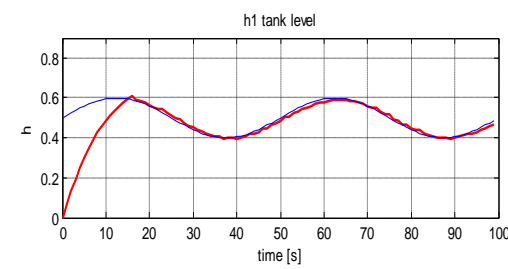

time [s]

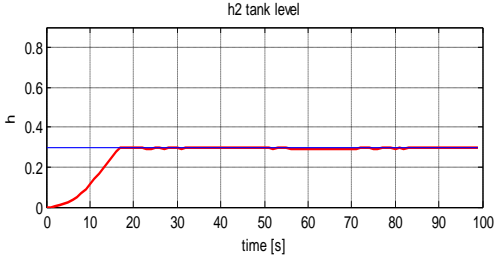

Fig 7: Liquid level in the two tank system In fact, the switching valves required to keep track of the reference, the states of the system remain close to the reference, and this objective is reached by a switching in the valves V2 and V4 Figure (5) and Figure 8. 


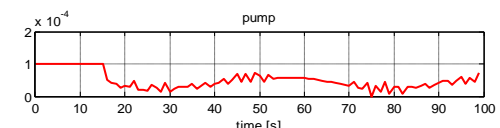

time $[s]$
VALVEV
S
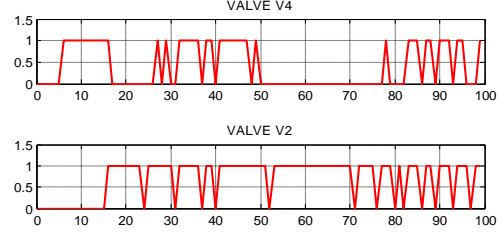

Fig 8. Continuous input Qp1, discrete inputs $V_{4}$ and $\mathbf{V}_{2}$

An actuator failure is considered on the valve V2 is blocked at $50 \mathrm{~s}$ in position off.

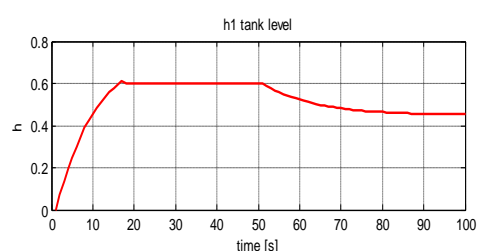
h2 tank level

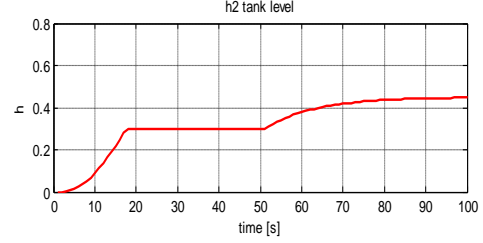

Fig 10: Liquid level in the two tank system Without reconfiguration MPC

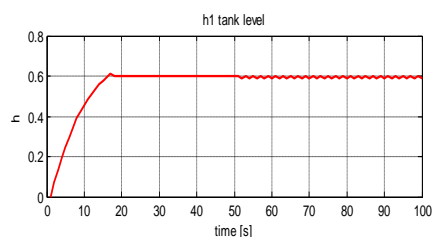

h2 tank levere

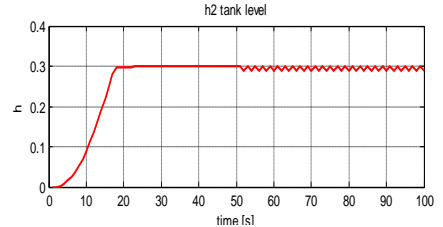

Figure 11: Liquid level in the two tank system With reconfiguration MPC

In fact the MPC constraints are updated based on the condition of the system by adding the constraint to present failure and using MLD failure. In this, case we show the faults tolerance capabilities of MPC controller figure 11 .
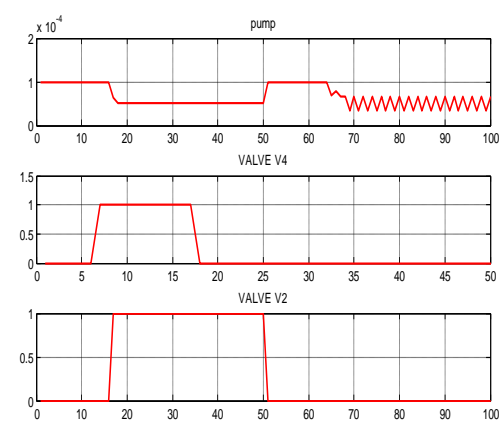

Fig12: Continuous input Qp1, discrete inputs V4 and V2

\section{CONCLUSION}

In this paper, we have developed and discussed new a methodology of active fault tolerant control based on Mixed Logical and Dynamical (MLD) we have adopted the model predictive control as a synthesis strategy. The design objectives and constraints are transformed into mixed-integer inequalities also the active fault tolerant problem is reformulated as a mixed integer optimization problem using the MLD framework.

When the fault is detected it is reconfiguration by updating the MPC constraints. Therefore the fault tolerance capabilities of MPC controller are proved. This strategy is finally applied in simulation to the level control of two tank benchmark.

\section{REFFRENCE}

[1] P.Antsaklis and X. Koutsoukos, "Hybrid systems: Review and recent progress," in Software-Enabled Control, T. Samad and G. Balas, Eds. IEEE Press, pp. 271-298, 2003.

[2] Y. Hao, Jiang, Bin, V. Cocquempot, "Fault Tolerant Control Design for Hybrid Systems" Series: Lecture Notes in Control and Information Sciences, (LNCIS). Edition Springer Verlag Vol. 397, 2010

[3] S. Narasimhan and G. Biswas, "Model-based diagnosis of hybrid systems," IEEE transactions on man and cybernetics, vol. 37, no. 3, pp. 347-361, 2007.

[4] J.Lunze, "Diagnosis of quantized systems by means of timed discrete-event representations," in Hybrid systems: Computation and Control, ser. Lecture Notes in Computer Science, N. Lynch and B. Krogh, Eds., vol. 1790. new york: Springer, pp. 258-27, 2000.

[5] F. Zhao, X. Koutsoukos, H. Haussecker, J. Reich, and P. Cheung, "Monitoring and fault diagnosis of hybrid

systems," IEEE Transactions on Systems, Man, and Cybernetics vol. 6, pp. 1225-1240, 2005.

[6] R. Mohammadi, S. Hashtrudi-Zad, and K. Khorasani, "A hybrid architecture for diagnosis in hybrid systems with applications to spacecraft propulsion system," in IEEE International Conference on Systems, Man and Cybernetics, pp. 3184-3190, 2007. 
[7] G. Ferrari-Trecate, D. Mignone, and, M. Morari. "Moving horizon estimation for hybrid systems." IEEE Transactions on Automatic Control 47 (10), pp 1663-1676. 2002.

[8] D. Mignone, A. Bemporad, and M. Morari, "A Framework for Control, Fault Detection, State Estimation, and Verification of Hybrid Systems" Proceedings of the American Control Conference, San Diego, pp. 13 Transactions on Automatic Control 47 (10), 1663-1676 2002.

[9] A.Bemporad and. M.Morari "Control of System Integrating Logic, Dynamics, and Constraints", Automatica 35(3), 407-427, 1999.

[10] Williams, H. P "Linear and integer programming applied to the propositional calculus, 2007.

[11] International Journal of Systems Research and Information Science vol 2. (1987).

[12] [Maciejowski J. Predictive "Control with Constraints”. Prentice-Hall: Great Britain, 2002.

[13] Brian Solberg, Palle Andersen, Jan M. Maciejowski Jakob Stoustrup Optimal switching control of burner setting for a compact marine boiler design journal Control Engineering Practice vol 18-pp 665675,2010 .
[14] M. Blanke, M. Kinnaert, J. Lunze, and M. Staroswiecki,"Diagnosis and Fault-Tolerant Control”. Springer- Verlag, 2006.

[15] D. Lefebvre, J.Ragot and al Diagnostic des SDH. "Quelques contributions issues des approches continues" Journal Européen des Systèmes Automatisés Vol 41 - pp.885-912, 2007.

[16] Y.Hammi, N. Zanzouri, M. Ksouri “ Model predictive control of hybrid systems with discrete and continuous inputs». International Conférence on Communications Computing and Control Applications, Hammamet, Tunis CCCA'1 2011.

[17] F.Torrisi and, A. Bemporad, "HYSDEL Modeling and Simulation of Hybrid Dynamical Systems". Proceedings of MATHMOD Conference, Vienna University of Technology, Vienna, Austria, February 5-7, 2003.

[18] F.D.Torrisi and A. Bemporad, "HYSDEL - a tool for generating computational hybrid models," IEEE Trans. Contr. Systems Technology, vol. 12, no. 2, pp. 235-249, 2004.

[19] A. Bemporad. Hybrid Toolbox for real time applications, User Guide, July 2010 